\title{
MISORIENTATION DEPENDENCE OF INTRINSIC GRAIN BOUNDARY MOBILITY: SIMULATION AND EXPERIMENT
}

\author{
M. UPMANYU ${ }^{1,2}$, D. J. SROLOVITZ ${ }^{2,3} \dagger$, L. S. SHVINDLERMAN $N^{4,5}$ and \\ G. GOTTSTEIN ${ }^{5}$ \\ 'Department of Materials Science and Engineering, University of Michigan, Ann Arbor, MI 48109- \\ 2136, U.S.A., ${ }^{2}$ Department of Mechanical and Aerospace Engineering, Princeton University, \\ Princeton, NJ 08544, U.S.A., ${ }^{3}$ Princeton Materials Institute, Princeton University, Princeton, \\ NJ 08544, U.S.A., ${ }^{4}$ Institut für Metallkunde und Metallphysik, RWTH Aachen, D-52056 Aachen, \\ Germany and ${ }^{5}$ Insitute of Solid State Physics, Russian Academy of Sciences, Chernogolovka, \\ Moscow distr. 142432, Russia
}

(Received 2 February 1999; accepted 25 June 1999)

\begin{abstract}
Both experimental and atomistic simulation measurements of grain boundary mobility were made as a function of temperature and boundary misorientation using the same geometry that ensures steady-state, curvature-driven boundary migration. Molecular dynamics simulations are performed using Lennard-Jones potentials on a triangular lattice. These simulations represent the first systematic study of the dependence of intrinsic grain boundary mobility on misorientation. The experiments focus on high purity $\mathrm{Al}$, with $\langle 111\rangle$ tilt boundaries, which are isomorphic to those examined in the simulations. Excellent agreement between simulations and experiments was obtained in almost all aspects of these studies. The boundary velocity is found to be a linear function of the curvature and the mobility is observed to be an Arrhenius function of temperature, as expected. The activation energies for boundary migration varies with misorientation by more than $40 \%$ in the simulations and $50 \%$ in the experiments. In both the simulations and experiments, the activation energies and the logarithm of the pre-exponential factor in the mobility exhibited very similar variations with misorientation, including the presence of distinct cusps at low $\Sigma$ misorientations. The activation energy for boundary migration is a logarithmic function of the pre-exponential factor in the mobility, within a small misorientation range around low $\Sigma$ misorientations. (C) 1999 Acta Metallurgica Inc. Published by Elsevier Science Ltd. All rights reserved.
\end{abstract}

Keywords: Grain boundaries; Computer simulation; Kinetics

\section{INTRODUCTION}

Most analyses of processes involving the motion of grain boundaries explicitly assume that grain boundary velocity $v$ is directly proportional to the driving force $F$ :

$$
v=M F
$$

where the constant of proportionality $M$ is the intrinsic grain boundary mobility (see, e.g. Ref. [1]). If we assume that boundary mobility is controlled by a single activated process, we should expect that the temperature dependence of the boundary mobility may be described using an Arrhenius relation

$$
M=M_{0} \mathrm{e}^{-Q / k_{\mathrm{B}} T}
$$

where $Q$ is the activation energy (enthalpy) for migration, $k_{\mathrm{B}}$ is Boltzmann's constant and $M_{0}$ is a (nearly) temperature independent pre-exponential factor [2]. In the case of curvature-driven grain boundary migration, where the driving force is associated with the decrease in the free energy of the

$\dagger$ To whom all correspondence should be addressed. system accompanying a reduction in grain boundary area, the rate of grain boundary migration is

$$
v=M \gamma \kappa
$$

where $\gamma$ is the grain boundary energy (per unit area) and $\kappa$ is the grain boundary curvature. While the curvature of the grain boundary is a purely geometrical variable, the mobility $M$ and the grain boundary energy $\gamma$ are intrinsic material parameters. The grain boundary mobility is known to vary by several orders of magnitude with variation in grain boundary crystallography within a single material [3-6], while the grain boundary energy rarely varies by more than a factor of two (neglecting twins and very low angle boundaries). Therefore, the key parameter determining the variation of grain boundary velocity within a given material is the variation in grain boundary mobility with boundary crystallography. Consequently, this variation in boundary mobility plays a key role in determining the evolution of crystallographic texture and grain boundary distribution during materials processing.

Over the past several decades, many experiments have been performed and theories developed to 
clarify our understanding of grain boundary mobility. Initially, grain growth experiments were conducted in polycrystalline samples to extract an average grain boundary mobility $\bar{M}$ from the relationship between grain size and time [7, 8]

$$
\bar{D}(t)^{2}-\bar{D}_{0}^{2} \propto \bar{M} t
$$

where $\bar{D}(t)$ is the average grain size at time $t, \bar{D}_{0}$ is the initial grain size and $\bar{M}$ is the average grain boundary mobility. However, since this process involved a statistical averaging over grain boundaries whose structure continuously changed during grain size evolution, the mobility data extracted from such experiments did not yield reliable information on the driving force or the misorientation dependence of the grain boundary mobility. The first experiments on individual grain boundaries were conducted by Aust and Rutter [3-5], who studied the effect of grain boundary misorientation and inclination on grain boundary migration in lead and lead-tin alloys. In these early experiments, grain boundary migration was induced by a wellcharacterized sub-grain (striation) structure in one of the grains meeting a flat boundary. These authors observed a non-monotonic variation of the activation energy for grain boundary migration with misorientation, with well-defined minima at several low sigma (coincidence site lattice) grain boundaries. Rath and $\mathrm{Hu}$ [9] used wedge-shaped bicrystals to experimentally extract grain boundary mobilities over a wide range of (curvature) driving forces in zone-refined aluminum. In these studies, the grain boundary curvature and velocity increased as the boundary migrated towards the apex of the wedge. Their non-steady-state data suggested a power law velocity-driving force relation: $v=M F^{m}$, where the exponent $m$ was in the range $1.5 \leq m \leq 12.3$ and was a function of the boundary misorientation. Sun and Bauer used the "reverse capillarity" technique in high purity $\mathrm{NaCl}$ bicrystals to extract grain boundary mobilities $[10,11]$. This experimental geometry resulted in a steadily decreasing driving force during the experiment. Sun and Bauer reported a linear relation between the velocity and the driving force over a limited range of driving forces and an Arrhenius-like temperature dependence of the mobility, albeit with a temperature dependent activation energy. In retrospect, these techniques clearly involve the motion of the grain boundary/sample edge contact lines, which can induce significant drag on grain boundary migration (either intrinsically or via groove dragging).

Constant integral curvature (hence constant driving force) migration experiments on single grain boundaries designed to yield steady-state grain boundary motion were first performed by Shvindlerman and co-workers $[6,12]$ in various high purity metals (including $\mathrm{Al}, \mathrm{Sn}$ and $\mathrm{Zn}$ ) using a half-loop grain bicrystal geometry (see Fig. 1), as

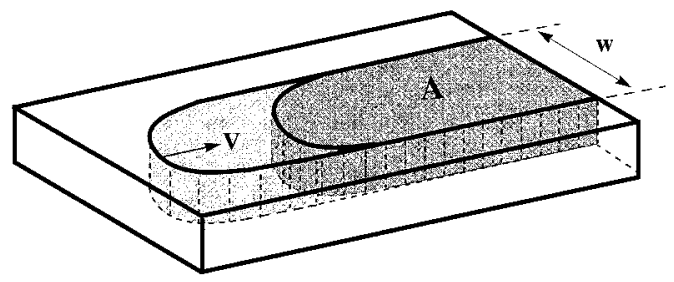

Fig. 1. The geometry used in both the simulations (twodimensional) and experimental U-shaped half-loop grain boundary migration study. The half-loop width is $w$, the surface area of the half-loop grain is $A$ and the velocity of the grain boundary at the base of the $\mathrm{U}$ is $v(=-\dot{A} / w)$.

well as a quarter-loop geometry. The quarter-loop geometry was first introduced by Dunn and coworkers [13] and employed by Aust et al. [14]. As the tip of the half-loop migrates, the sides of the grain boundary remain nearly parallel. Therefore, the integral curvature of the grain boundary remains constant throughout the migration process, leading to constant curvature/constant velocity boundary migration. Since our earlier experiments yielded a linear relationship between driving force and boundary velocity, we conclude that thermal groove drag effects are minimal [15].

The existence of a strong variation of impurity drag with the heat of impurity segregation, impurity concentration and boundary velocity makes it clear that there are (at best) few reliable experimental data on intrinsic grain boundary mobility. Determining the intrinsic boundary mobility and its dependence on boundary crystallography is key to the development of a predictive understanding of grain boundary motion. Once this is put on a firm foundation, it will be much easier to account for such complicated extrinsic effects such as those associated with impurities, defect absorption/generation, and contact lines. This realization has led several researchers to examine grain boundary migration from the perspective of atomistic computer simulations. These include the early work of Jhan and Bristowe [16] and Sutton [17], that did not lead to any predictions of boundary mobility. More recently, both Shönfelder et al. [18] and Upmanyu et al. [19] performed molecular dynamics simulations of the migration of grain boundaries with just a few misorientations and showed that the boundary velocity was indeed proportional to the driving force and that the temperature dependence of the boundary mobility was Arrhenius. However, these studies did not systematically examine the variation of grain boundary mobility with boundary crystallography.

The goal of the present paper is to systematically examine the variation of the intrinsic grain boundary mobility with grain boundary misorientation and temperature. In order to extract the essential el- 
ements of this dependence, special attention is focussed on the mobilities of boundaries with misorientations at or near several low sigma (high coincidence) misorientations and comparisons of these to general boundaries. The main approach employed in this study is molecular dynamics computer simulations with the half-loop bicrystal geometry employed with considerable success in the experiments of Shvindlerman and co-workers [6, 12, $20,22]$. The general trends in these results are directly compared with the experimental grain boundary mobility data. The plan of the present paper is to review the simulation and experimental approaches employed, demonstrate that the steadystate mobilities can be determined, and examine the temperature and misorientation dependence of boundary mobility. Comparisons between the simulation and experimental data are made throughout.

\section{SIMULATION AND EXPERIMENTAL APPROACHES}

The present simulations are designed to study steady-state, curvature-driven grain boundary migration. Grain boundary curvature-driven boundary migration was chosen for the present study because it is ubiquitous in material processing and other driving forces commonly entail modification of boundary structure (e.g. as in recrystallization). In addition, the most unambiguous experimental measurements of grain boundary mobility were performed using curvature-driven growth $[6,12,20$, 22]. In order to measure intrinsic, steady-state grain boundary migration, the simulation geometry must be chosen such that the driving force does not change during the migration process and such that there are no impurities or triple junctions to produce drag. Shvindlerman and co-workers [6, 12] introduced the U-shaped half-loop bicrystal geometry shown in Fig. 1 in order to study steady-state, curvature-driven boundary migration. This geometry is inherently two-dimensional in that the grain boundary traverses the entire thickness of the thin samples and remains (nearly) perpendicular to the surface during grain boundary migration. The curved section of the grain boundary has been observed to migrate with a self-similar shape [6], enabling the quantification of the grain boundary migration rate with the driving force (the curvature). In the experiments, the position of the grain boundary $\left(w \approx 5 \times 10^{-4} \mathrm{~m}\right)$ was followed using a continuous $\mathrm{X}$-ray tracking method which monitors the change in orientation of the crystals meeting at the grain boundary [20]. The position of the grain boundary was plotted as a function of time and the grain boundary migration rate is extracted from the time derivative of the grain boundary displacement-time curve. Care was taken to ensure that the migration of the grain boundary is observed in an "unloaded" regime, i.e. when the grain boundary is not pinned by an impurity cloud. Unfortunately, it is not possible to conclude that there was no effect of impurity drag even in the free motion regime of grain boundary displacement. Assuming that this motion was indeed free migration of the grain boundary, the reduced mobility of the grain boundary $M^{*}$ can be calculated from the extracted migration rate as

$$
M^{*}=M \gamma=(M \gamma)\left(\frac{\kappa}{2} w\right)=(M \gamma \kappa)\left(\frac{w}{2}\right)=-\frac{v w}{2}
$$

where we relate the curvature to the width of the U-shaped half-loop $w$ : namely $\kappa=2 / w$.

Simulations in this study are performed in two dimensions using the molecular dynamics simulation method and simple, empirical (LennardJones) pair potentials. We employ the same Ushaped, half-loop bicrystal geometry used by Shvindlerman and co-workers and constrain the sample to lie entirely in the $X-Y$ plane (see Fig. 1), as described in Ref. [6]. Three edges of the simulation cell are left free, so as to decrease the stresses produced during grain boundary migration. Three atomic planes are frozen on the fourth edge to maintain the desired grain misorientations. The simulations were all performed at constant temperature. The underlying crystal structure is that of a triangular lattice with a nearest neighbor spacing $r_{0}$. For more details, see Ref. [19]. Times are reported in units of $\tau$, energies in units of the Lennard-Jones potential well depth, distance in units of the equilibrium atom separation $r_{0}$, area in units of the perfect crystal area per atom $a_{0}$ and times in units of $\tau=\left(M_{\mathrm{at}} r_{0}^{2} / \varepsilon\right)^{1 / 2}$, where $M_{\mathrm{at}}$ is the atomic mass. The simulations were performed with bicrystal misorientations corresponding to various general (e.g. $\left.\Sigma=\infty-30^{\circ}\right)$, singular $\left(\Sigma=7-38.22^{\circ}\right.$ and $\left.13-32.23^{\circ}\right)$ and vicinal (near $\Sigma=7$ and 13) boundaries. The present study focuses on high angle boundaries, the misorientations examined here are limited to the range $30^{\circ}<\theta<40^{\circ}$ (the entire range of unique boundary misorientations is $30^{\circ}<\theta<60^{\circ}$ or, equivalently, $0^{\circ}<\theta<30^{\circ}$ ). The rate of change of the area of the half-loop grain, is simply $\dot{A}=v w$ (see Fig. 1), assuming a steady-state half-loop shape. If the migration is curvature driven, we may write $\dot{A}$ as

$$
\dot{A}=v w=-(M \gamma) \kappa w=-(M \gamma)\left(\frac{2}{w}\right) w=-2 M \gamma
$$

Hence, we extract the reduced mobilities from the rate of change of the area of the half-loop grain

$$
M^{*}=M \gamma=-\frac{\dot{A}}{2} \text {. }
$$

This equation implies that the reduced mobility is simply minus a half of the rate of change of the area of the half-loop grain and is independent of half-loop width. In order to determine the instan- 
taneous area of the half-loop grain, each atom is assigned to one of the two grains based upon the angles between it and its nearest neighbors (i.e. atoms nearer than $\sqrt{2} r_{0}$ ) relative to a fixed direction in the laboratory frame. The assignment to each grain is made based on whether the average nearest neighbor angles (modulo $2 \pi / 6$ ) are closer to those of the lattices interior to or exterior to the half-loop boundary. Unless otherwise noted, all data are averaged over at least three simulation runs.

\section{STEADY-STATE BEHAVIOR}

The temporal evolution of the atomic configuration of the half-loop computational cell is shown in Fig. 2 at $T=0.175 \varepsilon / k, w=25 r_{0}$ and $\theta=28.5^{\circ}$. Following an initial, short time transient, the asconstructed boundary equilibrates and establishes a profile [Fig. 2(a)] which remains self-similar throughout the migration process [cf. Figs 2(a) and (b)]. The evolution of the area of this half-loop $A$ with time $t$ is shown in Fig. 3. The main trend in

(a)

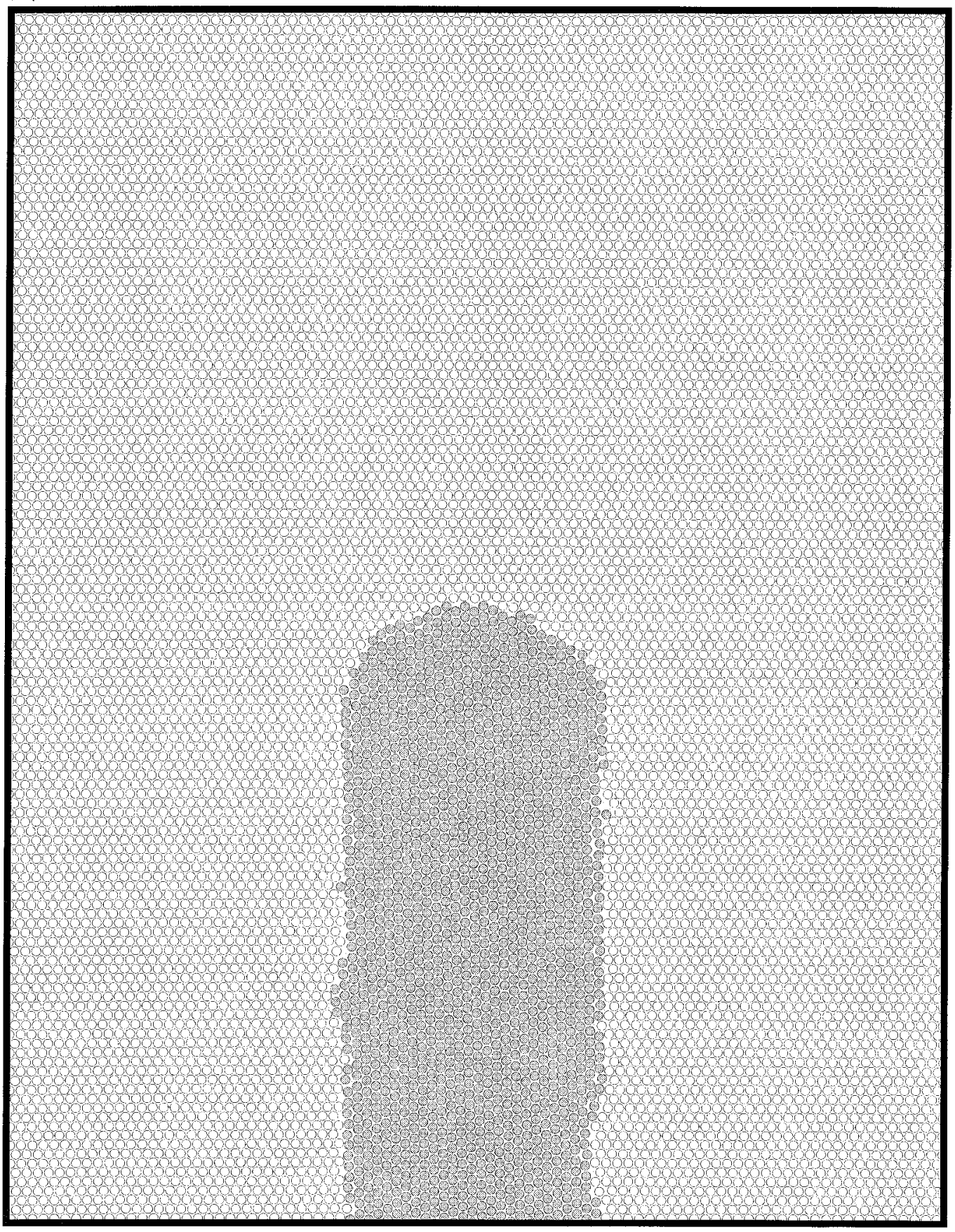

Fig. 2. (continued opposite) 
this figure is for the area of the half-loop grain to decrease linearly with time. The undulations in this curve are primarily normal thermal fluctuations associated with the atomic nature of the boundary migration process. The linearity of $A$ vs $t$ demonstrates the steady-state nature of the grain boundary migration during half-loop retraction. The slope of this linear plot yields the rate of change of area of the U-shaped half-loop, $\dot{A}$. In some cases, the steady-state retraction of the half-loop is interrupted by periods in which the boundary migration is significantly slowed. This is commonly due to the formation and emission of vacancies from the grain boundary as the system tries to eliminate the excess

(b)

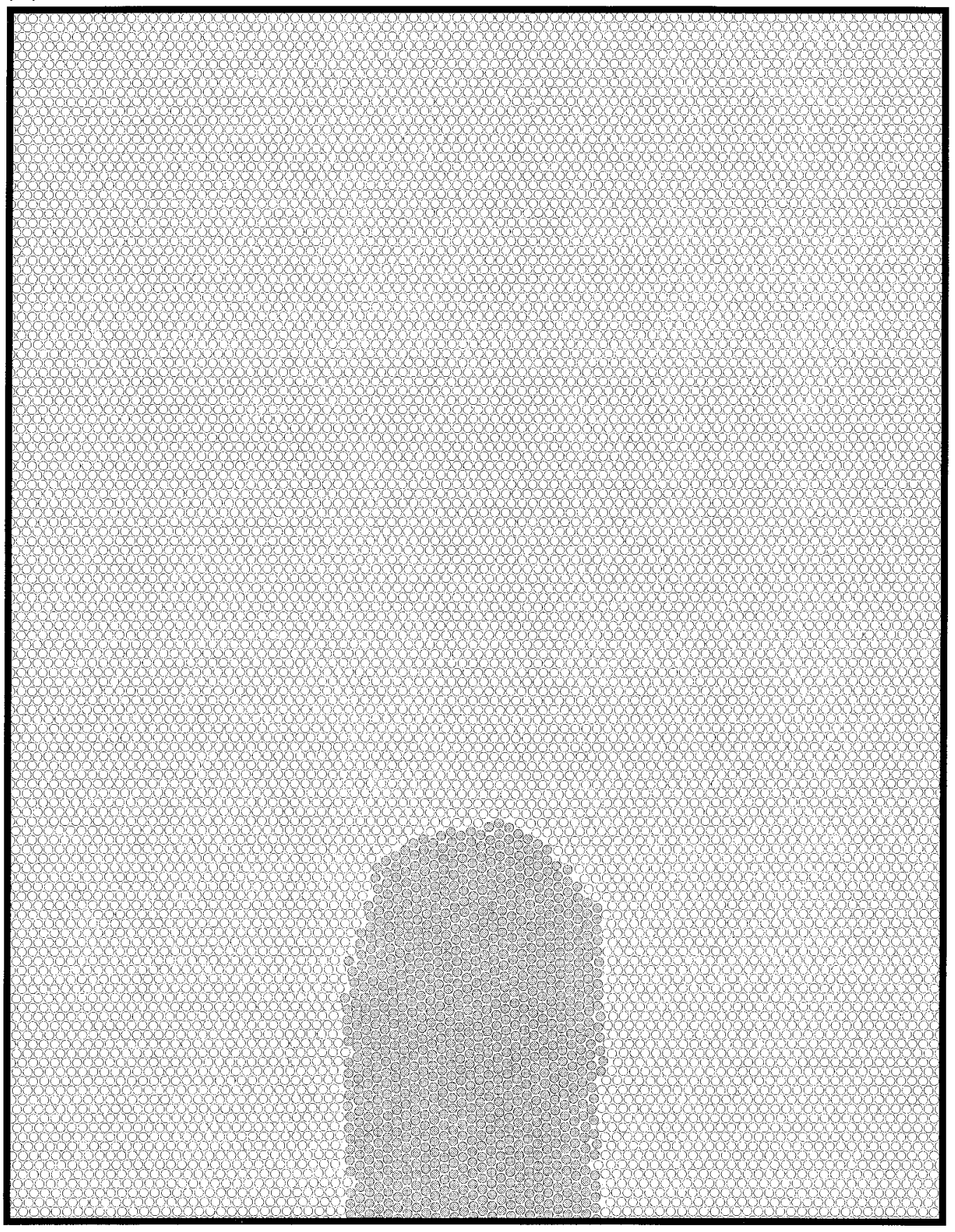

Fig. 2. Two atomic resolution images of the simulated retracting half-loop at different times: (a) $t=50 \tau$; (b) $t=860 \tau$. In this case, $T=0.175 \varepsilon / k, w=25 r_{0}$ and $\theta=28.5^{\circ}$. Note the similarity between the half-loop shapes. 


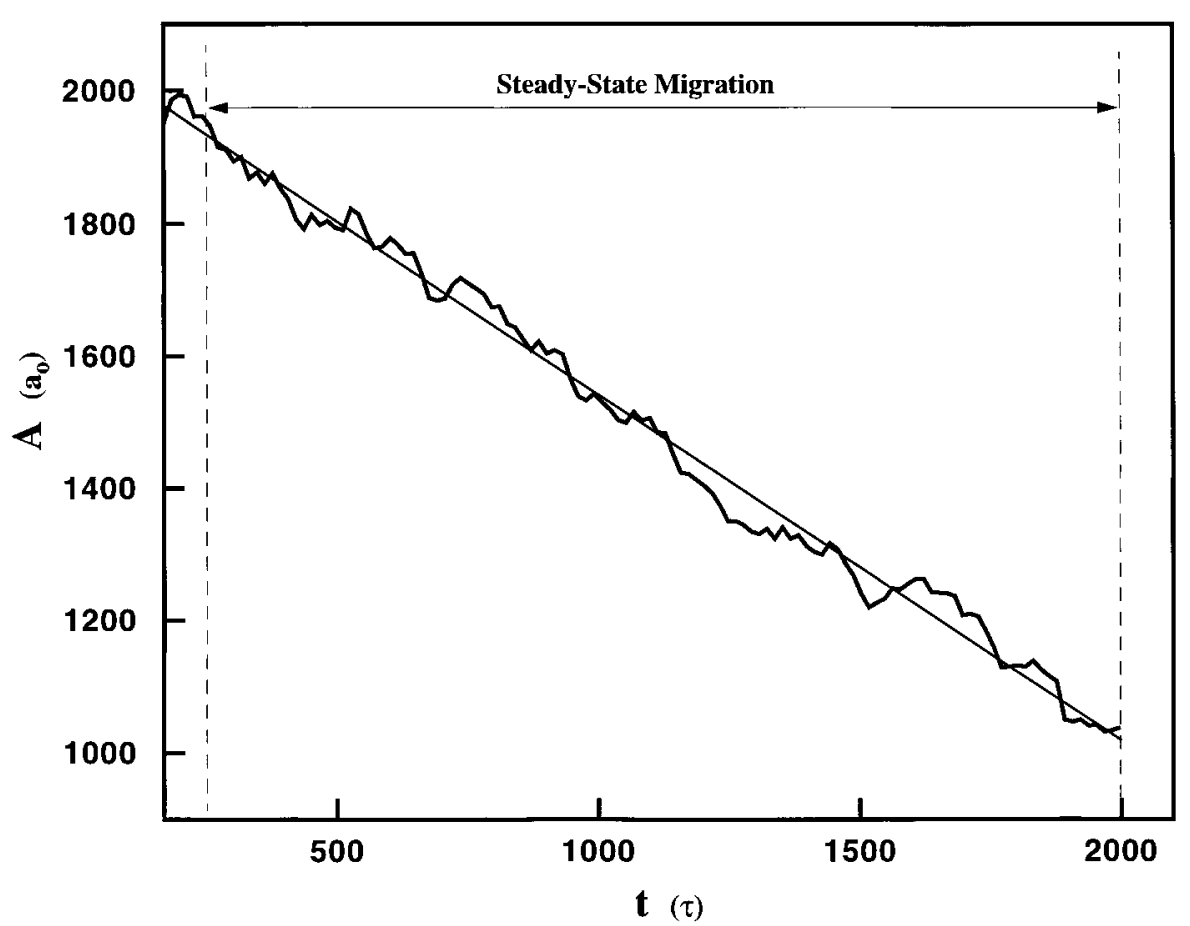

Fig. 3. Variation of the simulated area of the half-loop grain shown in Fig. $2\left(T=0.175 \varepsilon / k, w=25 r_{0}\right.$ and $\theta=28.5^{\circ}$ ) with time.

grain boundary volume associated with a decrease in the total length (area) of the grain boundary (see Ref. [21] for details). In such cases, the $\dot{A}$ data are taken at times following such vacancy emission events, as observed in images of the atomic configurations.

Self-similar migration of U-shaped half-loops and quarter-loops has also been directly observed in the experiments, where X-ray interface tracking was used to identify the position of the grain boundary at various instants of time [20, 22]. Examples of the grain boundary displacement-time graph (half-loop area is proportional to displacement times width) obtained using the continuous X-ray interface tracking technique [20] in high purity $\mathrm{Al}$ are shown in Fig. 4 for $\langle 111\rangle$ tilt grain boundaries for a misorientation of $\theta=40.5^{\circ}$ at temperatures of $T=777$ and $831 \mathrm{~K}$. Just as in the simulations, the grain boundaries in the experiments migrate at constant rates. The small fluctuations observed in the experimental data in Fig. 4 are associated with the method in which the boundary position is detected. The migration rate $v$, extracted from the data taken at these two temperatures $(777$ and $831 \mathrm{~K}$ ) in Fig. 4 are $v=4.1$ and $51.7 \mu \mathrm{m} / \mathrm{s}$, respectively.

The dependence of the grain boundary velocity on the driving force for boundary migration is determined by performing a series of simulations at a fixed temperature $(T=0.125 \varepsilon / k)$ for different half-loop widths (i.e. different curvatures). Since $\dot{A}$ is proportional to the reduced mobility $M^{*}=M \gamma$ [equation (7)], these data can be used to determine the curvature dependence of the boundary mobility. Figure 5 shows the boundary velocity $v(=\dot{A} / w)$ and the reduced mobility as a function of the grain boundary curvature $\kappa(=2 / w)$. At small curvatures (large half-loop widths), the grain boundary velocity is a linear function of the grain boundary curvature, as expected for curvature-driven growth [equation (6)]. Consequently, the reduced mobility $M^{*}$ is independent of curvature [see equations (6) and (7)] in this low curvature regime. These results confirm the assumption that the velocity is indeed proportional to the grain boundary curvature [equation (3)] and that the boundary mobility is independent of the magnitude of the driving force. In the present simulations, we find that $\dot{A}_{\infty}=-0.26\left(r_{0}\right)^{2} / \tau$, which corresponds to a reduced mobility of $M^{*}=M \gamma=0.13\left(r_{0}\right)^{2} / \tau$.

When the grain boundary curvature becomes very large (i.e. $w$ becomes small), the grain boundary velocity shows strong deviations from a linear dependence on boundary curvature (Fig. 5) and the grain boundary velocity exceeds our expectations based upon equation (6). Deviations from pure curvature-driven boundary migration are only significant for a half-loop width below 20r . This is presumably attributable to elastic interactions between different sections of the grain boundary, non-dissipative types of boundary motion at high velocity and/or discreteness effects associated with the atomic scale grain boundary profile [19]. These data suggest that it is possible to extract meaningful grain boundary mobilities from these types of simu- 


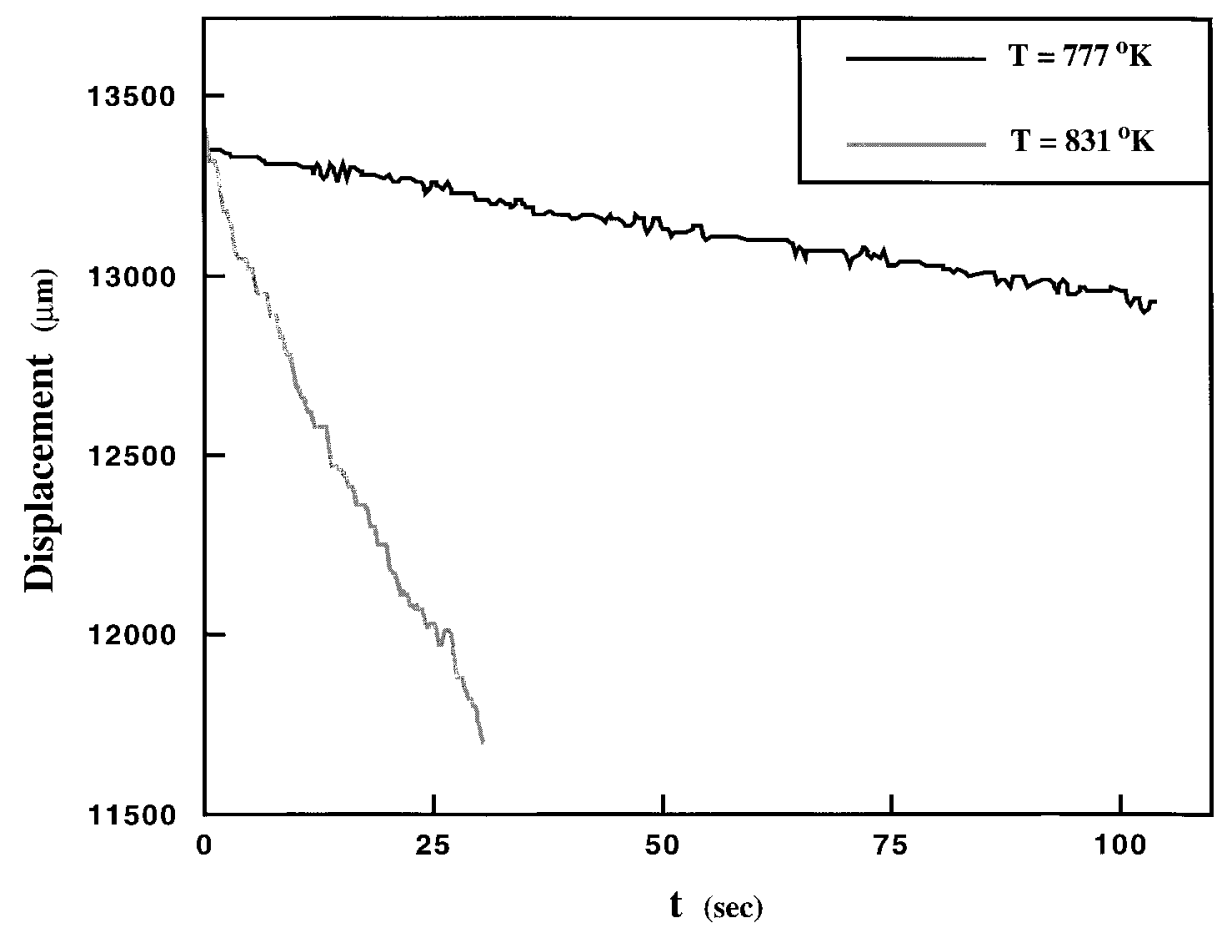

Fig. 4. Displacement of the top of the quarter-loop grain with time for two experiments performed in high purity $\mathrm{Al}$ at 777 and $831 \mathrm{~K}$ with a misorientation of $38.2^{\circ}$.

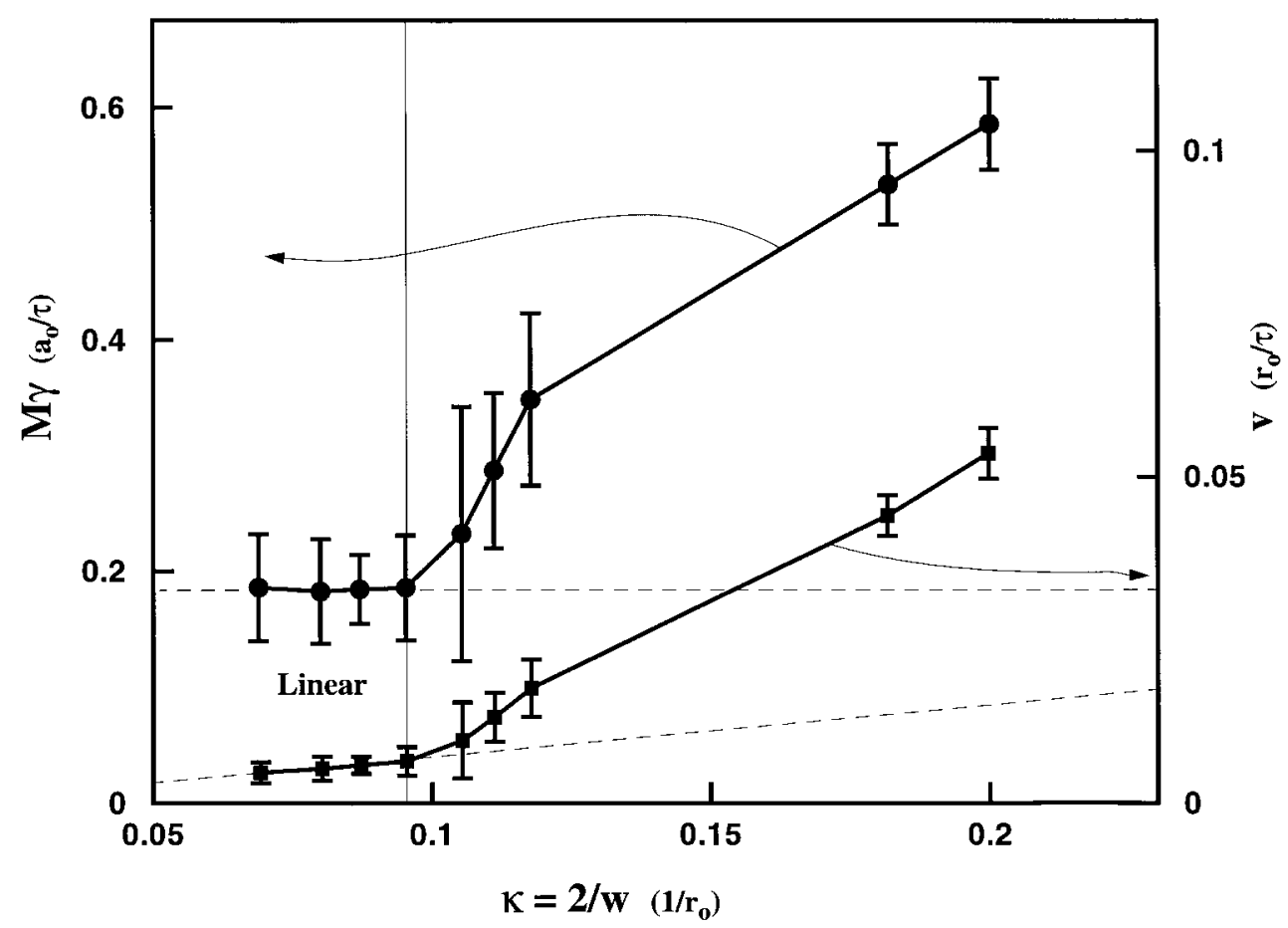

Fig. 5. Simulation results showing both the reduced mobility (left side) and boundary velocity (right side) vs curvature. For curvatures less than approximately $0.1 / r_{0}$, the boundary velocity is a linear function of curvature and the reduced mobility is independent of curvature. Substantial deviations from this behavior are observed at large curvatures. The reduced mobility curve is shifted upwards by $0.05 a_{0} / \tau$. 
lations provided that the half-loop width is sufficiently large (i.e. above approximately $20 r_{0}$ ).

The derivative of the experimental displacementtime graphs (e.g. Fig. 4) with respect to time provides an experimental measure of the velocity $v$ of the grain boundary migration. Figure 6 presents experimental data on the variation of the velocity of grain boundary migration with driving force $\kappa / \gamma$ for U-shaped half-loops [18] (normalized by the interface energy) for $\langle 111\rangle$ tilt grain boundaries in high purity $\mathrm{Al}$ at a constant temperature. Examination of this plot shows that the grain boundary velocity is a linear function of the driving force for sufficiently high driving forces, in agreement with the atomistic simulation results. At small grain boundary curvatures, Fig. 6 shows that the grain boundary velocity is slower than expected. This is presumably attributable to impurity drag effects at low velocities.

The data in this section clearly demonstrate that experiment and atomistic simulation show excellent correspondence with each other and that steadystate grain boundary migration is achieved using the U-shaped half-loop bicrystal geometry (provided that the half-loop width is sufficiently large and impurity drag effects are negligible). Both experiment and simulation clearly demonstrate, that grain boundary velocity is proportional to grain boundary curvature.

\section{TEMPERATURE DEPENDENCE OF MOBILITY}

The temperature dependence of the reduced grain boundary mobility is determined by performing a series of simulations over a temperature range from $T=0.075 \varepsilon / k$ to $0.250 \varepsilon / k$ and with a half-loop width $w$ for which classical curvature-driven growth occurs, $w=25 r_{0}$. The simulations were performed for boundary misorientations corresponding to singular (low $\Sigma$ ), vicinal (near singular) and general grain boundaries. Figure 7 shows the variation of the logarithm of the reduced mobility $M \gamma$ vs inverse temperature for $\Sigma=13\left(\theta=32.21^{\circ}\right)$, near $\Sigma=13$ $\left(\theta=33^{\circ}, 31.5^{\circ}\right), \quad \Sigma=7 \quad\left(\theta=38.23^{\circ}\right), \quad$ near $\quad \Sigma=7$ $\left(\theta=38.98^{\circ}, 37.51^{\circ}\right)$ and $\Sigma=\infty \quad\left(\theta=30^{\circ}\right)$. These data sets are each well fitted by a straight line $\left(\ln M^{*}=\ln M_{0}^{*}-Q / k_{\mathrm{B}} T\right)$, the slope of which yields the activation energy for grain boundary migration $\left(-Q / k_{\mathrm{B}}\right)$ and the $y$-intercept $(1 / T=0)$ yields the pre-exponential factor for the intrinsic reduced mobility, for each grain boundary. The data in Table 1 show that the activation energy for these grain boundaries lies between $0.3 \varepsilon$ and $0.5 \varepsilon$, where $\varepsilon$ is the bond strength (i.e. the well depth of the Lennard-Jones pair potential). The linearity of the data (on the axes of Fig. 7) demonstrates that the mobility is indeed an Arrhenius function of temperature [as per equation (2)], at least in the temperature range examined $(Q / k T \gg 1)$. This is

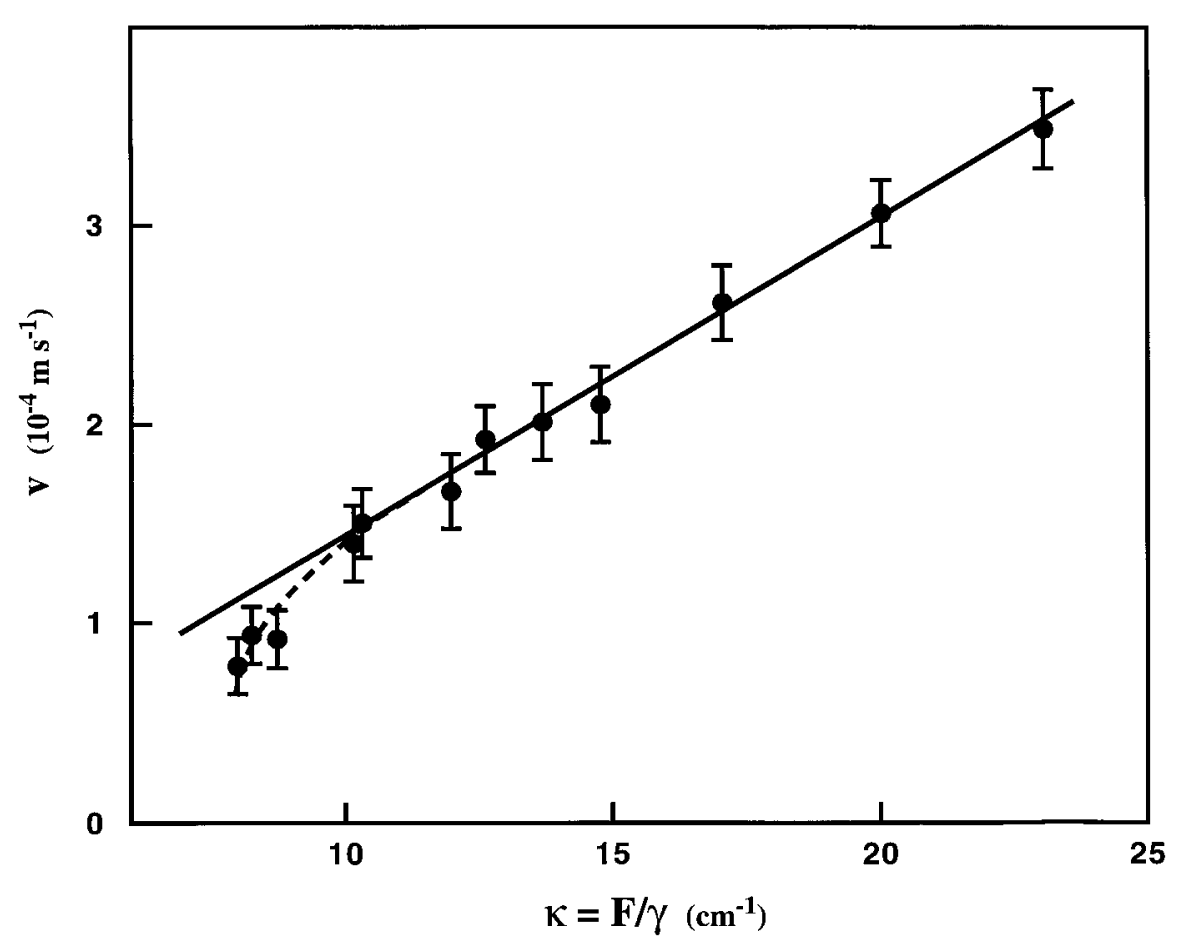

Fig. 6. Experimental measurements of grain boundary velocity vs boundary curvature for a general grain boundary in high purity $\mathrm{Al}$. 


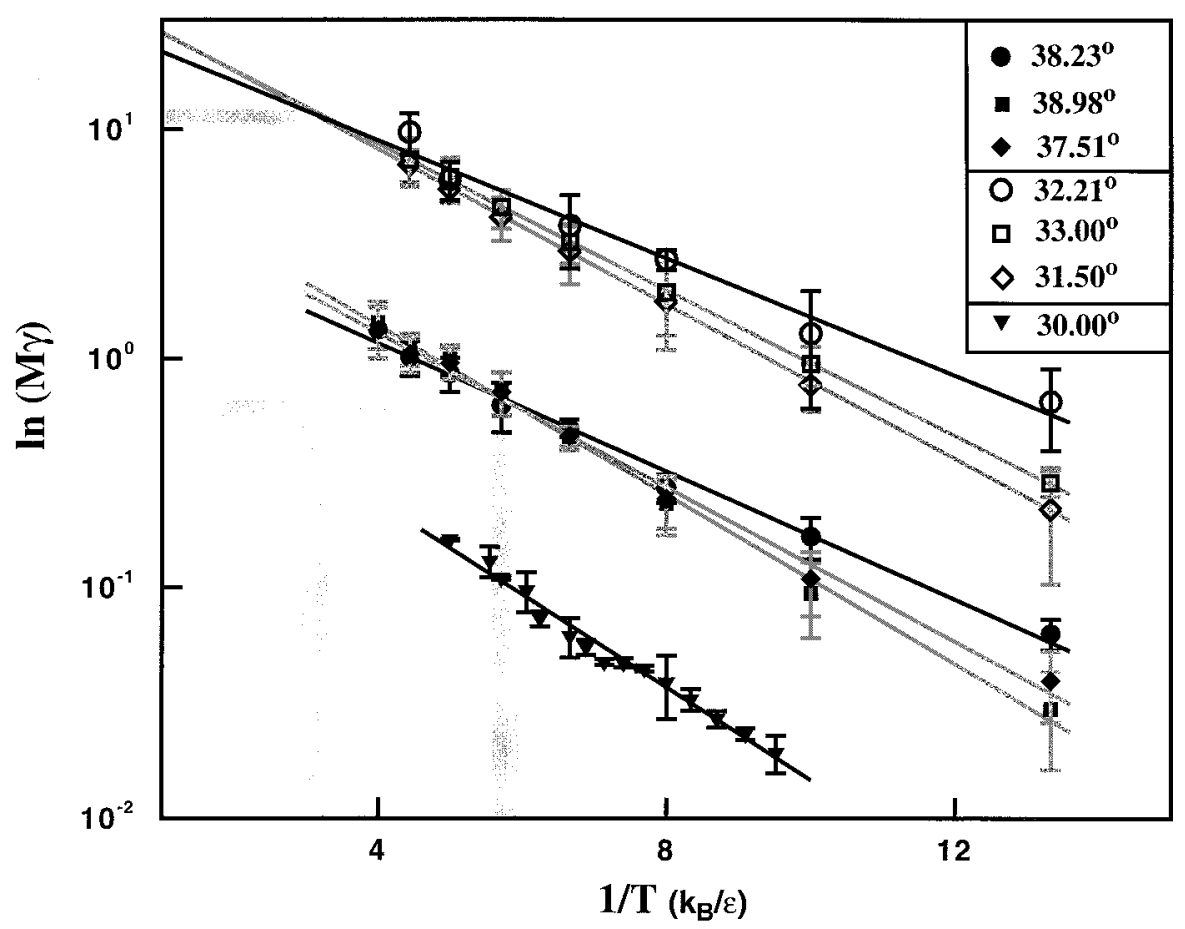

Fig. 7. Arrhenius plots of reduced mobility (logarithm of reduced mobility vs inverse temperature) for a series of simulations corresponding to misorientations at or near $\Sigma=7$ (filled circles, squares and diamonds), at or near $\Sigma=13$ (open symbols) and $\Sigma=\infty$ (i.e. $\theta=30^{\circ}$, inverted, filled triangles). The $\Sigma=7$ series data are shifted upwards by $\ln (2)$ and the $\Sigma=\infty$ curve is shifted downwards by $\ln (1 / 8)$ for clarity.

consistent with the predictions of absolute reaction rate theory.

Figure 8 shows the experimentally determined reduced mobilities for $\langle 111\rangle$ tilt grain boundaries in high purity $\mathrm{Al}\left(\theta=38.2^{\circ}\right.$ and $\left.40.5^{\circ}\right)$ vs inverse temperature in the same form as the simulation data (Fig. 7). The reduced mobilities for both boundaries are well fitted by straight lines, yielding activation energies of 1.25 and $2.2 \mathrm{eV}$, respectively. The linearity of the two data sets in Fig. 8 demonstrate that the reduced grain boundary mobility is Arrhenius with temperature, confirming the results obtained through computer simulations above and the ansatz in equation (3).

It is not possible to make accurate direct comparisons between the simulation and experimental data (e.g. activation energies for boundary migration) because of the inaccuracies of the interatomic potentials and the different crystal structures in the two cases. Nonetheless, crude comparisons are possible based on scaling the parameters in the interatomic potential and similarities between the crystallography. While the experimental study focuses on $\langle 111\rangle$ tilt grain boundaries in a face centered cubic material $(\mathrm{Al})$, the simulations are performed on a two-dimensional triangular lattice. Since the two-dimensional triangular lattice is isostructural with $\{111\}$ planes, the grain boundaries are closely (but by no means completely) equivalent. We can relate the experimental measurements of the activation energies to the simulation results by normalizing the experimental results by the effective bond energy, $\varepsilon$ : i.e. the cohesive energy of the solid $E_{\text {coh }}$ divided by half the number of nearest neighbor atoms. Using the experimental value for the activation energy for the migration of a $\Sigma=7$ grain boundary in $\mathrm{Al}$ [22] and normalizing it by the bond energy $(\varepsilon)$, we find $Q / \varepsilon=2.2$. The corresponding value from the simulations is $Q / \varepsilon=0.30$. The agreement between the two is poor, with the experimental value 7.3 times larger than that predicted by the simulations. A similar discrepancy was observed by Shönfelder et al. when comparing

Table 1. The activation energies and pre-exponential factors for reduced mobilities for various misorientations extracted from the performed simulations

\begin{tabular}{lcc}
\hline$\theta\left({ }^{\circ}\right)$ & $Q(\varepsilon)$ & $\ln \left(M_{0} \gamma\right)$ \\
\hline 40.000 & $0.456 \pm 0.013$ & $17.4 \pm 2.2$ \\
39.500 & $0.410 \pm 0.033$ & $18.1 \pm 2.6$ \\
38.976 & $0.365 \pm 0.021$ & $18.0 \pm 3.7$ \\
$38.213(\Sigma=7)$ & $0.302 \pm 0.039$ & $14.8 \pm 3.5$ \\
37.517 & $0.390 \pm 0.030$ & $18.9 \pm 4.8$ \\
37.000 & $0.429 \pm 0.044$ & $20.2 \pm 3.2$ \\
35.567 & $0.468 \pm 0.033$ & $18.4 \pm 3.0$ \\
33.992 & $0.456 \pm 0.020$ & $18.2 \pm 3.4$ \\
33.000 & $0.435 \pm 0.031$ & $14.6 \pm 2.9$ \\
$32.204(\Sigma=13)$ & $0.319 \pm 0.020$ & $7.5 \pm 1.3$ \\
32.000 & $0.358 \pm 0.037$ & $10.1 \pm 1.9$ \\
31.500 & $0.393 \pm 0.015$ & $12.1 \pm 2.8$ \\
31.000 & $0.425 \pm 0.027$ & $12.5 \pm 3.3$ \\
$30.000(\Sigma=\infty)$ & $0.466 \pm 0.025$ & $15.5 \pm 0.6$ \\
\hline
\end{tabular}




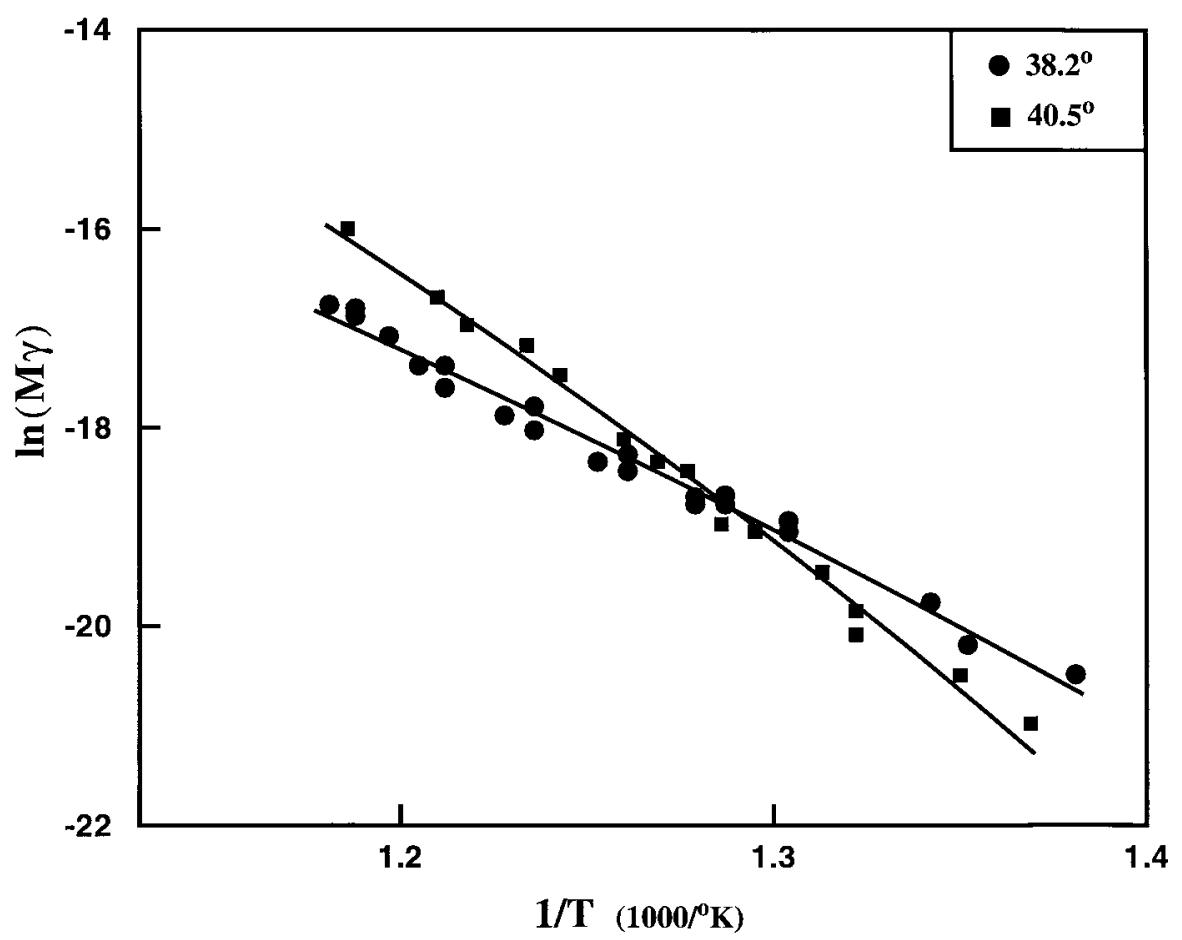

Fig. 8. As in Fig. 7, but for experimental data on $\mathrm{Al}$ for misorientations of $\theta=38.2^{\circ}(\Sigma=7)$ and $40.5^{\circ}$.

three-dimensional molecular dynamics simulation of $\langle 100\rangle$ flat twist boundary motion to experimental results [18].

This much larger experimental activation energy for boundary migration is likely attributable to impurity drag. Even though the purity of the Al in these experiments is very high impurity, the total impurity content at or near the grain boundary can still be quite substantial. If the distribution of impurities in the material are indeed modified by the grain boundary, the activation energy for grain boundary migration can be as large as the sum of the bulk impurity diffusivity plus the impurityboundary binding energy. The activation energy for diffusion of a typical impurity $(\mathrm{Mn})$ in $\mathrm{Al}$, is $2.2 \mathrm{eV}$ [23] or $Q / \varepsilon=1.7$, which differs from the experimental activation energy for boundary migration by $0.5 \varepsilon(0.65 \mathrm{eV})$ and represents a plausible impurityboundary binding energy. There is a large body of evidence which shows that decreasing impurity concentrations decreases the activation energy for boundary migration [3-6]. The fact that the present simulations are two-, rather than three-dimensional, suggests that the boundary structures and the nature of the activated states in the simulation and the experiments may be substantially different. There is no doubt that the activated state is more constrained in two dimensions, compared with three. This should lead to higher activation energies and, hence, does not resolve the discrepancy. The Lennard-Jones potentials used in the present simulations are known to be too stiff in compression to describe metals. This too would be expected to yield activation energies that are too high (in contradiction to the present results). Therefore, our preliminary conclusion is that the discrepancy between simulation and experimental determination of the intrinsic grain boundary mobility is primarily attributable to impurity drag effects in the experiments (i.e. the experiments are not measuring intrinsic boundary mobility).

\section{MISORIENTATION DEPENDENCE OF MOBILITY}

A series of simulations was performed for misorientations in the range of $30^{\circ}<\theta<40^{\circ}$ (corresponding to singular, vicinal and general grain boundaries) and for temperatures in the range from $T=0.075 \varepsilon / k$ to $0.250 \varepsilon / k$ (half-loop width $\left.w=25 r_{0}\right)$. These data were tabulated in the form of Fig. 7 and fitted to obtain the activation energy for boundary migration and the pre-exponential factor for the reduced mobility $M_{0}^{*}$ (Table 1$)$. The variations of the activation energy and the pre-exponential factor with misorientation are shown in Fig. 9. Clearly neither $Q$ nor $M_{0}^{*}$ vary monotonically with misorientation. The activation energy vs misorientation plot shows two pronounced minima (cusps) at misorientations corresponding to $\Sigma=13$ $\left(\theta=32.20^{\circ}\right)$ and $\Sigma=7\left(\theta=38.23^{\circ}\right)$. The variation of the pre-exponential factor $M_{0}^{*}$ for the reduced mobility also shows distinct minima (cusps) at the same misorientations $\left(\Sigma=13, \quad \theta=32.20^{\circ}\right.$ and $\Sigma=7, \theta=38.23^{\circ}$ ). The variation of $M_{0}^{*}$ with $\theta$ is much less smooth than the $Q$ vs $\theta$ plot, however to within the error bars, each curve shows smooth 
variation with $\theta$ away from the cusps. The similarities between the variation of $Q$ and $\ln \left(M_{0} \gamma\right)$ with $\theta$ suggests that the pre-exponential factor and activation energy for boundary migration are positively correlated. This is discussed in the next section.

The variation of the activation energy and the pre-exponential factor (for the reduced mobility) with misorientation of $\langle 111\rangle$ tilt grain boundaries in high purity $\mathrm{Al}$ has been extracted from the experimental data and is shown in Fig. 10. As in the simulations (Fig. 9), this variation is not monotonic, with minima in both $Q$ and $M_{0}^{*}$ occurring at or near $\theta=38.2^{\circ}$ (i.e. near $\Sigma=7$ ). No experimental data near $\Sigma=13\left(\theta=32.20^{\circ}\right)$ are available for comparison with the simulation results. As in the case of the simulation study, the misorientations for which maxima and minima in the activation energy vs misorientation curves are observed are replicated in the pre-exponential curves. This is experimental confirmation of the close correlation between $Q$ and $\ln \left(M_{0} \gamma\right)$ observed in the simulations. Similarly, the misorientation dependence of the grain boundary mobility in $\mathrm{Al}$ is much more pronounced at low temperature then at higher temperature, as in the simulations.
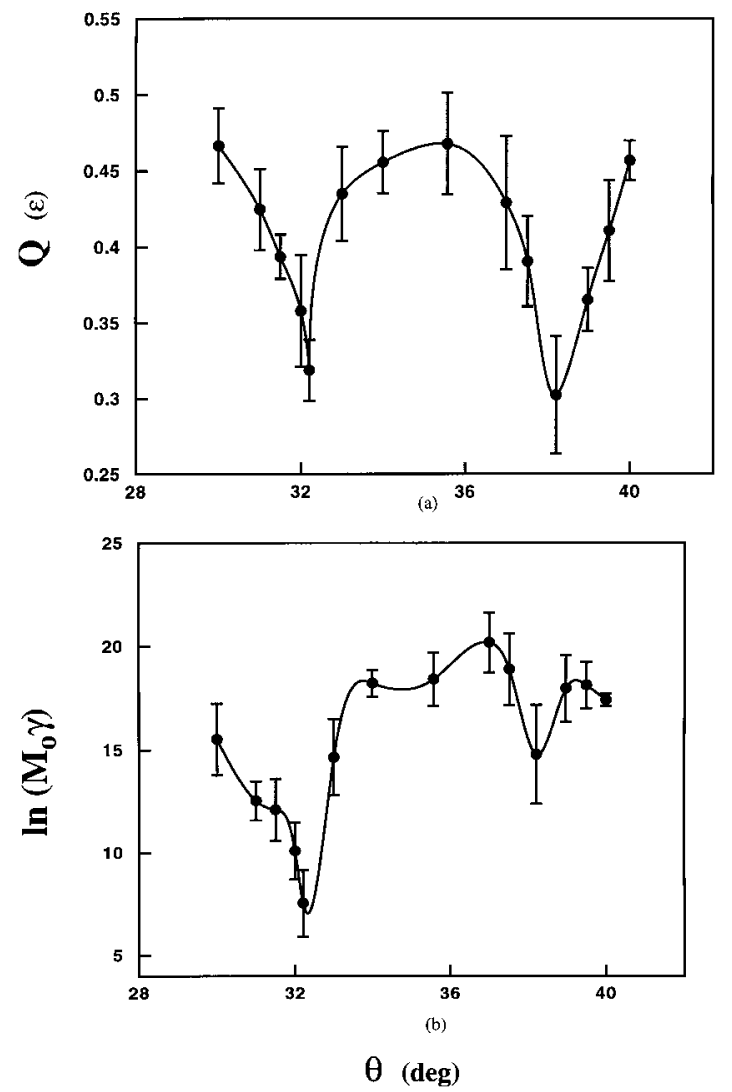

Fig. 9. The activation energy for boundary migration (a) and the logarithm of the pre-exponential factor (b) of the reduced mobility in the simulations as a function of boundary misorientation. These data are tabulated in Table 1 .
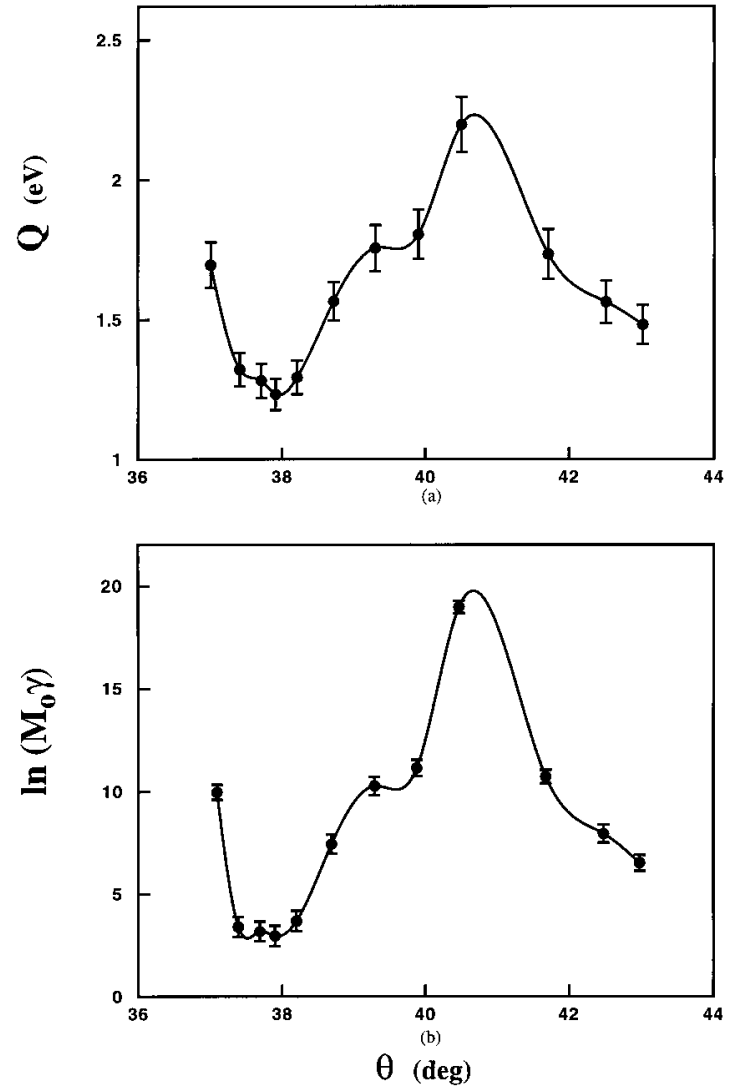

Fig. 10. Same as Fig. 9, but for the experimental data on Al.

Examination of the reduced mobility data in Figs 7 and 8 shows that the magnitude of the variation of the mobility with misorientation is temperature dependent. Assuming that the mobility is an Arrhenius function of temperature, we find that the temperature dependence of the variation of the mobility with respect to misorientation

$$
\frac{\partial \ln M^{*}}{\partial \theta}=\frac{\partial \ln M_{0}^{*}}{\partial \theta}-\frac{1}{k_{\mathrm{B}} T} \frac{\partial Q}{\partial \theta}
$$

is controlled by the misorientation dependence of the activation energy alone. Therefore, the misorientation dependence of the mobility should be more pronounced at low temperatures than at high temperatures, as is borne out by Fig. 7 and Tables 2 and 3. Equation (8) also suggests that there is a specific temperature at which the variation of the mobility with misorientation is exactly zero (i.e. the mobility is misorientation independent). This too is discussed in the next section.

\section{COMPENSATION EFFECT}

The simulation and experimental results presented in the previous section suggest that the activation energy and the logarithm of the reduced mobility vary in the same manner with changes in boundary misorientation or, therefore, boundary 
Table 2. The reduced mobilities [in units of $\left(a_{0}^{2}\right) /\left(\tau r_{0}\right)$ from simulations performed for four different grain boundaries compared at two different temperatures

\begin{tabular}{|c|c|c|c|c|}
\hline & $\Sigma=13\left(\theta=32.20^{\circ}\right)$ & $\theta=30.00^{\circ}$ & $\theta=33.99^{\circ}$ & $\Sigma=7\left(\theta=32.20^{\circ}\right)$ \\
\hline$T=0.125 \varepsilon / k\left(\approx 0.3 T_{\mathrm{m}}\right)$ & $0.274 \pm 0.038$ & $0.155 \pm 0.048$ & $0.392 \pm 0.039$ & $0.682 \pm 0.067$ \\
\hline$T=0.200 \varepsilon / k\left(\approx 0.5 T_{\mathrm{m}}\right)$ & $0.865 \pm 0.148$ & $0.649 \pm 0.005$ & $1.014 \pm 0.124$ & $1.515 \pm 0.285$ \\
\hline
\end{tabular}

Table 3. The experimentally extracted reduced mobilities (in units of $\mathrm{m}^{2} / \mathrm{s}$ ) for three different boundaries, compared at two different temperatures

\begin{tabular}{lccc}
\hline & $\theta=38.2^{\circ}(\Sigma=7)$ & $\theta=42.0^{\circ}$ & $\theta=40.50^{\circ}$ \\
\hline$T=633 \mathrm{~K}\left(\approx 0.7 T_{\mathrm{m}}\right)$ & $2.5 \times 10^{-9}$ & $8 \times 10^{-10}$ & $2.5 \times 10^{-10}$ \\
$T=753 \mathrm{~K}\left(\approx 0.8 T_{\mathrm{m}}\right)$ & $9.5 \times 10^{-8}$ & $1.5 \times 10^{-7}$ & $8.0 \times 10^{-8}$ \\
\hline
\end{tabular}

structure. In order to quantify this relationship we plot $\ln \left(M_{0} \gamma\right)$ vs $Q$ (Fig. 11). These data clearly show that a relationship of the form

$$
Q=\alpha \ln \left(M_{0} \gamma\right)+\beta
$$

exists for boundaries of similar misorientation, where $\alpha$ and $\beta$ are constants. In particular, we find that the data point for the $\Sigma=7, \theta=38.21^{\circ}$ boundary and those for the boundaries that are only slightly misoriented from it (i.e. its vicinal boundaries, $37^{\circ} \leq \theta \leq 39^{\circ}$ ) accurately lie on one line (one set of $\alpha$ and $\beta$ ), while the data point for the $\Sigma=13, \quad \theta=32.2^{\circ}$ boundary and those for the boundaries that are only slightly misoriented from it $\left(31.5^{\circ} \leq \theta \leq 33^{\circ}\right)$ accurately lie on a different straight line. The boundaries that are outside of these angular ranges are widely distributed and fall on neither line. The fact that the data for the near $\Sigma=7$ and near $\Sigma=13$ boundaries fall onto distinct lines suggest that the underlying structure of these two sets of boundaries is quite distinct.

Figure 12 shows the same type of plot as in Fig. 11 [i.e. $Q$ vs $\ln \left(M_{0} \gamma\right)$ ] for the experimental data on $\Sigma=7$ and near $\Sigma=7\langle 111\rangle$ tilt grain boundaries in high purity $\mathrm{Al}$. As in the simulation results, the experimental data all fall on a single straight line.

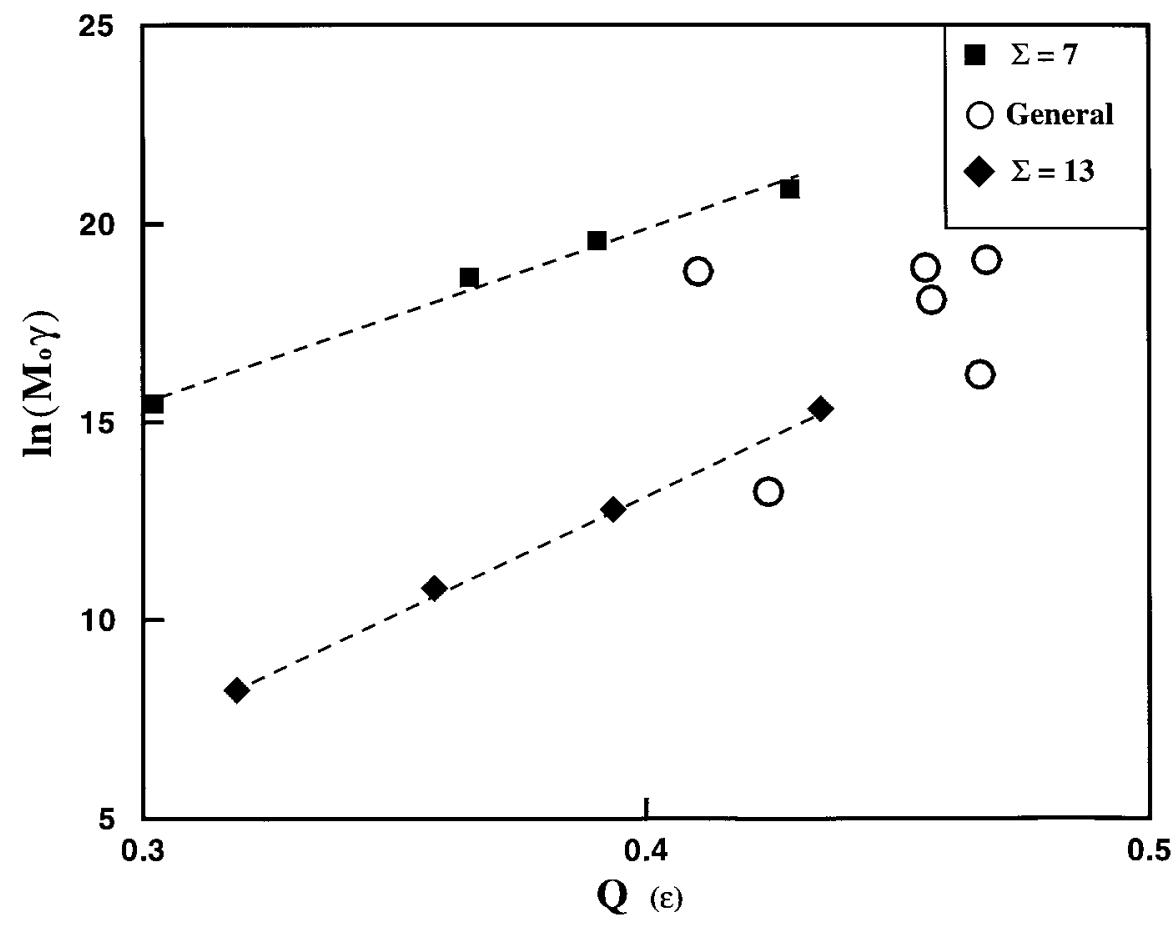

Fig. 11. Logarithm of the pre-exponential factor of the reduced mobility vs the activation energy for boundary migration for a range of misorientations for the simulation data presented in Fig. 9 (error bars indicated in Table 1). The data plotted as filled squares correspond to the boundaries with misorientations at or near $\Sigma=7\left(38.32^{\circ}, 38.97^{\circ}, 37.51^{\circ}, 37.00^{\circ}\right)$. The data plotted as filled diamonds correspond to the boundaries with misorientations at or near $\Sigma=13\left(32.20^{\circ}, 32.00^{\circ}, 31.50^{\circ}\right.$ and $\left.33.00^{\circ}\right)$. The open circles correspond to misorientations outside these ranges. 


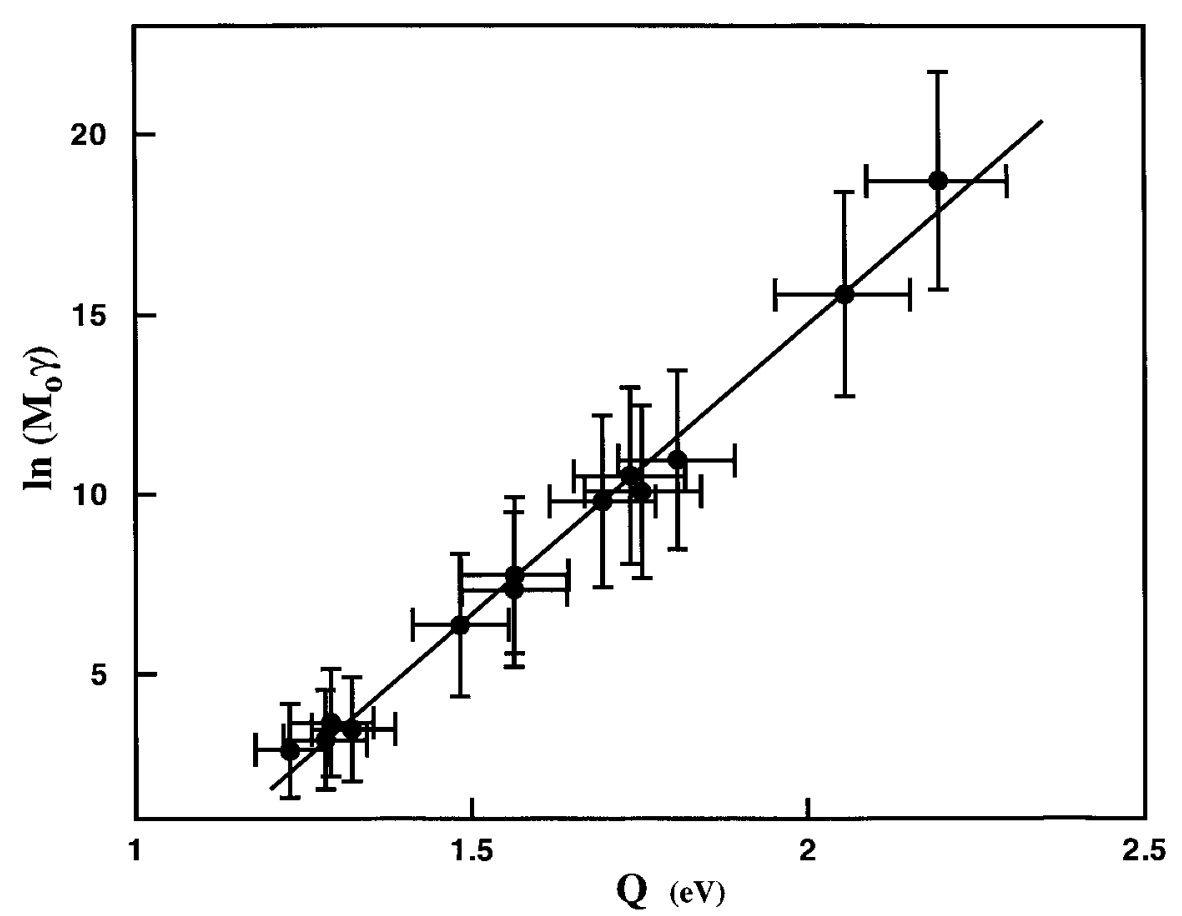

Fig. 12. Same as Fig. 11, but for the experimental Al data presented in Fig. 10.

These experimental and atomistic simulation data, taken in toto, clearly demonstrate that the variation of the pre-exponential factor and activation energy for grain boundary mobility amongst structurally related boundaries is well described by the relationship in Fig. 10. This same relationship was obtained earlier [20] based upon a wide range of grain boundary mobility experimental measurements (different materials, different rotation axes).

The linear relation between the activation energy and the logarithm of the pre-exponential factor describing grain boundary mobility, represented by equation (9), shows that an increase in activation energy (which tends to decrease the boundary mobility) will be compensated by a concomitant increase in the pre-exponential factor (which tends to increase the boundary mobility). This implies that the mobilities of structurally related boundaries will be much closer than the differences in activation energy would normally suggest. This is indeed the case, as the data in Figs 7 and 8 show. This effect has become known as the compensation effect, the thermodynamic and kinetic origin of which is discussed in Ref. [24].

The existence of the compensation effect [as represented by equation (9)], and the functional form of the mobility [equation (2)], implies that there exists a compensation temperature, $T_{\mathrm{c}}$, at which the mobilities of these closely related grain boundaries are all equal. Solving equations (2) and (9) for two different boundaries and setting them equal implies that $T_{\mathrm{c}}=\alpha / k_{\mathrm{B}}$. Extraction of the values of $\alpha$ from the simulation data in Fig. 11 implies that $T_{\mathrm{c}}=$ $\alpha / k_{\mathrm{B}}=0.40 \pm 0.02 \varepsilon / k_{\mathrm{B}}$ for the near $\Sigma=7$ boundaries and $T_{\mathrm{c}}=\alpha / k_{\mathrm{B}}=0.192 \pm 0.018 \varepsilon / k_{\mathrm{B}}$ for the near $\Sigma=13$ boundaries. This is very close to that obtained from the crossing of the mobilities (simulation) vs inverse temperature curves in Fig. 7: from $T_{\mathrm{c}}=0.38 \varepsilon / k_{\mathrm{B}}$ to $0.41 \varepsilon / k_{\mathrm{B}}$ for the near $\Sigma=7$ boundaries and from $T_{\mathrm{c}}=0.180 \varepsilon / k_{\mathrm{B}}$ to $0.200 \varepsilon / k_{\mathrm{B}}$ for the near $\Sigma=13$ boundaries. The experimental data for the near $\Sigma=7$ boundaries in Al in Fig. 12 imply $T_{\mathrm{c}}=\alpha / k_{\mathrm{B}}=719 \mathrm{~K}$ and the temperature where the two curves in Fig. 8 cross is $T_{\mathrm{c}}=770 \mathrm{~K}$. The accuracy to which the values of $T_{\mathrm{c}}$ are obtained using the two approaches (simulation $\sim 1 \%$, experiment $\sim 7 \%$ ) is an indication of the accuracy to which the Arrhenius form of the mobility [equation (2)] and equation (9) fit the data. Given the uncertainty in the experimental and simulation data, this is excellent agreement.

\section{CONCLUSIONS}

We have made extensive measurements of grain boundary mobility as a function of temperature and boundary misorientation in a geometry where boundary migration is driven by grain boundary curvature. Both molecular dynamics simulations using Lennard-Jones potentials on a triangular lattice and experiments on $\langle 111\rangle$ tilt boundaries in high purity Al were performed. These simulations 
represent the first systematic study of the dependence of intrinsic grain boundary mobility on misorientation. In both experiments and simulations, steady-state grain boundary migration was achieved. The boundary velocity was found to be a linear function of the curvature, as expected. Plots of the logarithm of the mobility vs inverse temperature were linear; indicating that the temperature dependence of the mobility is Arrhenius. The same plots showed that the activation energies varied by more than $40 \%$ in the simulations and $50 \%$ in the experiments with changes in misorientation. The mobilities of boundaries in a small misorientation range around low $\Sigma$ misorientations became the same at a well-defined temperature, $T_{\mathrm{c}}$ (that varied from one low $\Sigma$ misorientation to another). In both the simulations and experiments, the activation energies and the logarithm of the pre-exponential factor in the mobility exhibited very similar variations with misorientation, including the presence of distinct cusps at low $\Sigma$ misorientations. This is a manifestation of the compensation effect. The activation energy for boundary migration was shown to be a logarithmic function of the pre-exponential factor in the mobility, within a small misorientation range around low $\Sigma$ misorientations. This explains the observation that grain boundary mobility often does not vary as drastically with misorientation as the activation energies themselves would suggest and the observed equality of boundary mobilities at a well-defined temperature. Aside from a discrepancy in the absolute value of the activation energy, we find that overall excellent agreement between the simulation and experimental data was obtained. Such a discrepancy in the absolute values is to be expected when comparing two-dimensional simulations using oversimplified interatomic potentials with real experiments.

Acknowledgements-M.U. and D.J.S. gratefully acknowledge the financial support of the Division of Materials Science of the Office of Basic Energy Sciences of the United States Department of Energy Grant \#FG0288ER45367, under whose auspices this research was performed. L.S.S. and G.G. express their gratitude to the Deutsche Forschungsgemeinschaft and to the Russian Foundation for Fundamental Research under contract $N$ 96-02-17483 for financial support for their collaboration.

\section{REFERENCES}

1. Humphreys, F. J. and Hatherly, M., Recystallization and Related Annealing Phenomena. Pergamon Press, Oxford, 1996, Chap. 4.

2. Turnbull, D., Trans. Am. Inst. Min. Engrs, 1951, 191, 661.

3. Aust, K. T. and Rutter, J. W., Trans. Am. Inst. Min. Engrs, 1959, 215, 119.

4. Aust, K. T. and Rutter, J. W., Trans. Am. Inst. Min. Engrs, 1959, 215, 820.

5. Aust, K. T. and Rutter, J. W., Acta metall., 1965, 13, 181.

6. Aristov, V. Y., Fridman, Y. M. and Shvindlerman, L. S., Physics Metals Metallogr., 1973, 35, 859.

7. Gordon, P. and Vandermeer, R. A., Recrystallization, Grain Growth and Textures. Am. Soc. of Metals, Cleveland, OH, 1966.

8. Holmes, E. and Winegard, W. C., Can. J. Phys., 1959 , 37, 469.

9. Rath, B. B. and Hu, H., Trans. Am. Inst. Min. Engrs, 1969, 245, 1577.

10. Sun, R. C. and Bauer, C. L., Acta metall., 1970, 18 , 635.

11. Sun, R. C. and Bauer, C. L., Acta metall., 1970, 18 639.

12. Aristov, V. Y., Fradkov, V. E. and Shvindlerman, L. S., Soviet Phys. Solid St., 1980, 22, 1055.

13. Dunn, C. G., Daniels, F. W. and Bolton, M. J., Trans. Am. Inst. Min. Engrs, 1949, 185, 785.

14. Aust, K. T., Harrison, R. J. and Maddin, R., J. Inst Metals, 1956, 85, 57.

15. Gottstein, G. and Shvindlerman, L. S., Scripta metall. mater., 1992, 27, 1521.

16. Jhan, R. J. and Bristowe, P. D., Scripta metall. mater., 1990, 24, 1313.

17. Sutton, A. P., in Computer Simulation in Materials Science: Nano/Meso/Macroscopic Space and Time Scales, ed. H. O. Kirchner, L. P. Kubin and V. Pontikis. NATO-ASI Series E, Vol. 308, 1996, p. 163.

18. Shönfelder, B., Wolf, D., Philpot, S. R. and Furtkamp, M., Interface Sci., 1997, 5, 245.

19. Upmanyu, M., Smith, R. W. and Srolovitz, D. J., Interface Sci., 1998, 6, 41.

20. Gottstein, G., Molodov, D. A. and Shvindlerman, L. S., Interface Sci., 1998, 6, 41.

21. Upmanyu, M., Srolovitz, D. J., Gottstein, G. and Shvindlerman, L., Interface Sci., 1998, 6(4), 289.

22. Molodov, D. A., Czubayko, U., Gottstein, G. and Shvindlerman, L. S., Scripta metall. mater., 1995, 32, 529.

23. Brandes, E. A. (ed.), Smithells Metals Reference Book, 6th edn. Butterworths, London, 1983, pp. 13-15.

24. Gottstein, G. and Shvindlerman, L. S., Interface Sci., 1998, 6(4), 267. 